\title{
The Foreground of Big Bang Nucleosynthesis
}

\author{
Ravi Karki \\ Department of Physic, PN Campus, Pokhara, Email: ravikarkinp@yahoo.com
}

In physical cosmology, Big Bang nucleosynthesis (or primordial nucleosynthesis, BBN) refers to the production of nuclei other than those of $\mathrm{H}-1$ (i.e. the normal, light isotope of hydrogen, whose nuclei consist of a single proton each) during the early phases of the universe. Primordial nucleosynthesis took place just a few minutes after the Big Bang and is believed to be responsible for the formation of a heavier isotope of hydrogen known as deuterium (H-2 or D), the helium isotopes He-3 and He-4, and the lithium isotopes Li-6 and Li-7. In addition to these stable nuclei some unstable, or radioactive, isotopes were also produced during primordial nucleosynthesis: tritium or $\mathrm{H}-3$; beryllium-7 (Be-7), and beryllium-8 (Be-8). These unstable isotopes either decayed or fused with other nuclei to make one of the stable isotopes.

\section{Characteristics of Big Bang nucleosynthesis}

There are two important characteristics of Big Bang nucleosynthesis $(\mathrm{BBN})$ :

It lasted for only about seventeen minutes (during the period from 3 to about 20 minutes from the beginning of space expansion after that, the temperature and density of the universe fell below that which is required for nuclear fusion. The brevity of BBN is important because it prevented elements heavier than beryllium from forming while at the same time allowing unburned light elements, such as deuterium, to exist.

It was widespread, encompassing the entire observable universe. The key parameter which allows one to calculate the effects of BBN is the number of photons per baryon. This parameter corresponds to the temperature and density of the early universe and allows one to determine the conditions under which nuclear fusion occurs. From this we can derive elemental abundances. Although the baryon per photon ratio is important in determining elemental abundances, the precise value makes little difference to the overall picture. Without major changes to the Big Bang theory itself, BBN will result in mass abundances of about $75 \%$ of $\mathrm{H}-1$, about $25 \%$ helium- 4 , about $0.01 \%$ of deuterium, trace (on the order of $10^{-10}$ ) amounts of lithium and beryllium, and no other heavy elements. (Traces of boron have been found in some young stars, giving rise to the question that some boron, not really predicted by the theory, might have been produced in the Big Bang. The question is not presently resolved. That the observed abundances in the universe are generally consistent with these abundance numbers is considered strong evidence for the Big Bang theory.In this field it is customary to quote percentages by mass, so that $25 \%$ helium- 4 means that helium- 4 atoms account for $25 \%$ of the mass, but only about $8 \%$ of the atoms would be helium-4 atoms.

\section{Sequence of Big Bang nucleosynthesis}

Big Bang nucleosynthesis begins about three minutes after the Big Bang, when the universe has cooled down sufficiently to form stable protons and neutrons, after baryogenesis. The relative abundances of these particles follow from simple thermodynamical arguments, combined with the way that the mean temperature of the universe changes over time (if the reactions needed to reach the thermodynamically favoured equilibrium values are too slow compared to the temperature change brought about by the expansion, abundances will remain at some specific non-equilibrium value). Combining thermodynamics and the changes brought about by cosmic expansion, one can calculate the fraction of protons and neutrons based on the temperature at this point. The answer is that there are about seven protons for every neutron at the beginning of nucleogenesis, a ratio that would remain stable even after nucleogenesis is over. This fraction is in favour of protons initially primarily because lower mass of the proton favors their production. Free neutrons also decay to protons with a half-life of about 15 minutes, and this time- 
scale is too short to affect the number of neutrons over the period in which BBN took place, primarily because most of the free neutrons had already been absorbed in the first 3 minutes of nucleogenesis-- a time too short for a significant fraction of them to decay to protons.

One feature of BBN is that the physical laws and constants that govern the behavior of matter at these energies are very well understood, and hence BBN lacks some of the speculative uncertainties that characterize earlier periods in the life of the universe. Another feature is that the process of nucleosynthesis is determined by conditions at the start of this phase of the life of the universe, making what happens before irrelevant.

As the universe expands, it cools. Free neutrons and protons are less stable than helium nuclei, and the protons and neutrons have a strong tendency to form helium-4. However, forming helium-4 requires the intermediate step of forming deuterium. At the time at which nucleosynthesis occurs, the temperature is high enough for the mean energy per particle to be greater than the binding energy of deuterium; therefore any deuterium that is formed is immediately destroyed (a situation known as the deuterium bottleneck). Hence, the formation of helium-4 is delayed until the universe becomes cool enough to form deuterium (at about $\mathrm{T}=0.1 \mathrm{MeV}$ ), when there is a sudden burst of element formation. Shortly thereafter, at twenty minutes after the Big Bang, the universe becomes too cool for any nuclear fusion to occur. At this point, the elemental abundances are fixed, and only change as some of the radioactive products of BBN (such as tritium) decay.

\section{History of Big Bang nucleosynthesis theory}

The history of Big Bang nucleosynthesis began with the calculations of Ralph Alpher and George Gamow in the 1940s. Together with Hans Bethe they would publish the seminal Alpher-Bethe-Gamow paper outlining the theory of light-element production in the early universe.

During the 1970s, there was a major puzzle in that the density of baryons as calculated by Big Bang nucleosynthesis was much less than the observed mass of the universe based on calculations of the expansion rate. This puzzle was resolved in large part by postulating the existence of dark matter.

\section{Heavy elements}

Big Bang nucleosynthesis produced no elements heavier than beryllium, due to a bottleneck due to the absence of a stable nucleus with 8 or 5 nucleons. In stars, the bottleneck is passed by triple collisions of helium-4 nuclei, producing carbon (the triple-alpha process). However, this process is very slow, taking tens of thousands of years to convert a significant amount of helium to carbon in stars, and therefore it made a negligible contribution in the minutes following the Big Bang.

\section{Helium-4}

Big Bang nucleosynthesis predicts a primordial abundance of about 25\% helium-4 by mass, irrespective of the initial conditions of the universe. As long as the universe was hot enough for protons and neutrons to transform into each other easily, their ratio, determined solely by their relative masses, was about 1 neutron to 7 protons (allowing for some decay of neutrons into protons). Once it was cool enough, the neutrons quickly bound with an equal number of protons to form helium- 4 . Helium- 4 is very stable and neither decays nor combines easily to form heavier nuclei. So out of every 16 nucleons ( 2 neutrons and 14 protons), 4 of these $(25 \%)$ combined into one helium- 4 nucleus. One analogy is to think of helium-4 as ash, and the amount of ash that one forms when one completely burns a piece of wood is insensitive to how one burns it.

The helium- 4 abundance is important because there is far more helium-4 in the universe than can be explained by stellar nucleosynthesis. In addition, it provides an important test for the Big Bang theory. If the observed helium abundance is much different from $25 \%$, then this would pose a serious challenge to the theory. This would particularly be the case if the early helium-4 abundance was much smaller than $25 \%$ because it is hard to destroy helium- 4 . For a few years during the mid-1990s, observations suggested that this might be the case, causing astrophysicists 
to talk about a Big Bang nucleosynthetic crisis, but further observations were consistent with the Big Bang theory.

\section{Deuterium}

Deuterium is in some ways the opposite of helium-4 in that while helium-4 is very stable and very difficult to destroy, deuterium is only marginally stable and easy to destroy. Because helium- 4 is very stable, there is a strong tendency on the part of two deuterium nuclei to combine to form helium-4. The only reason BBN does not convert all of the deuterium in the universe to helium- 4 is that the expansion of the universe cooled the universe and cut this conversion short before it could be completed. One consequence of this is that unlike helium-4, the amount of deuterium is very sensitive to initial conditions. The denser the universe is, the more deuterium gets converted to helium- 4 before time runs out, and the less deuterium remains.

There are no known post-Big Bang processes which would produce significant amounts of deuterium. Hence observations about deuterium abundance suggest that the universe is not infinitely old, which is in accordance with the Big Bang theory.

During the 1970s, there were major efforts to find processes that could produce deuterium, which turned out to be a way of producing isotopes other than deuterium. The problem was that while the concentration of deuterium in the universe is consistent with the Big Bang model as a whole, it is too high to be consistent with a model that presumes that most of the universe consists of protons and neutrons. If one assumes that all of the universe consists of protons and neutrons, the density of the universe is such that much of the currently observed deuterium would have been burned into helium- 4 .

This inconsistency between observations of deuterium and observations of the expansion rate of the universe led to a large effort to find processes that could produce deuterium. After a decade of effort, the consensus was that these processes are unlikely, and the standard explanation now used for the abundance of deuterium is that the universe does not consist mostly of baryons, and that non-baryonic matter (also known as dark matter) makes up most of the matter mass of the universe. This explanation is also consistent with calculations that show that a universe made mostly of protons and neutrons would be far more clumpy than is observed.

It is very hard to come up with another process that would produce deuterium via nuclear fusion. What this process would require is that the temperature be hot enough to produce deuterium, but not hot enough to produce helium-4, and that this process immediately cools down to non-nuclear temperatures after no more than a few minutes. Also, it is necessary for the deuterium to be swept away before it reoccurs.

Producing deuterium by fission is also difficult. The problem here again is that deuterium is very subject to nuclear processes, and that collisions between atomic nuclei are likely to result either in the absorption of the nuclei, or in the release of free neutrons or alpha particles. During the 1970s, attempts were made to use cosmic ray spallation to produce deuterium. These attempts failed to produce deuterium, but did unexpectedly produce other light elements.

\section{Observational Tests and Status of Big Bang nucleosynthesis}

The theory of BBN gives a detailed mathematical description of the production of the light "elements" deuterium, helium-3, helium-4, and lithium-7. Specifically, the theory yields precise quantitative predictions for the mixture of these elements, that is, the primordial abundances.

In order to test these predictions, it is necessary to reconstruct the primordial abundances as faithfully as possible, for instance by observing astronomical objects in which very little stellar nucleosynthesis has taken place (such as certain dwarf galaxies) or by observing objects that are very far away, and thus can be seen in a very early stage of their evolution (such as distant quasars).

As noted above, in the standard picture of $\mathrm{BBN}$, all of the light element abundances depend on the amount 
of ordinary matter (baryons) relative to radiation (photons). Since the universe is homogeneous, it has one unique value of the baryon-to-photon ratio. For a long time, this meant that to test BBN theory against observations one had to ask: can all of the light element observations be explained with a single value of the baryon-to-photon ratio? Or more precisely, allowing for the finite precision of both the predictions and the observations, one asks: is there some range of baryon-to-photon values which can account for all of the observations?

More recently, the question has changed: Precision observations of the cosmic microwave background radiation with the Wilkinson Microwave Anisotropy Probe (WMAP) give an independent value for the baryon-to-photon ratio. Using this value, are the BBN predictions for the abundances of light elements in agreement with the observations?

The present measurement of helium- 4 indicates good agreement, and yet better agreement for helium-3 . But for lithium-7, there is a significant discrepancy between BBN and WMAP, and the abundance derived from Population II stars. The discrepancy is a factor of 2.4-4.3. and is considered a problem for the original models, that have resulted in revised calculations of the standard BBN based on new nuclear data, and to various reevaluation proposals for primordial proton-proton nuclear reactions, especially the intensities of ${ }^{7} \mathrm{Be}(\mathrm{n}, \mathrm{p})^{7} \mathrm{Li}$ versus ${ }^{7} \mathrm{Be}(\mathrm{d}, \mathrm{p}){ }^{8} \mathrm{Be}$.

\section{Non-standard Big Bang nucleosynthesis}

In addition to the standard BBN scenario there are numerous non-standard BBN scenarios. These should not be confused with non-standard cosmology: a non-standard BBN scenario assumes that the Big Bang occurred, but inserts additional physics in order to see how this affects elemental abundances. These pieces of additional physics include relaxing or removing the assumption of homogeneity, or inserting new particles such as massive neutrinos.

There have been, and continue to be, various reasons for researching non-standard $\mathrm{BBN}$. The first, which is largely of historical interest, is to resolve inconsistencies between $\mathrm{BBN}$ predictions and observations. This has proved to be of limited usefulness in that the inconsistencies were resolved by better observations, and in most cases trying to change $\mathrm{BBN}$ resulted in abundances that were more inconsistent with observations rather than less. The second, which is largely the focus of non-standard $\mathrm{BBN}$ in the early 21st century, is to use BBN to place limits on unknown or speculative physics. For example, standard BBN assumes that no exotic hypothetical particles were involved in $\mathrm{BBN}$. One can insert a hypothetical particle (such as a massive neutrino) and see what has to happen before BBN predicts abundances which are very different from observations. This has been usefully done to put limits on the mass of a stable tau neutrino. 